\title{
Abastecimiento energético de una casa tipo en la Patagonia Argentina
}

\section{Energy supply of a type house in the Argentine Patagonia}

Maximiliano Fernando Medina, María Eugenia De San Pedro, Hugo Miguel Chacon, Horacio Leonardo Leon, Carlos Ramiro Rodriguez, Abdelbassat Abdelbaki maximiliano.f.medina@gmail.com,edesanpedro@uaco.unpa.edu.ar Universidad Nacional de la Patagonia Austral - Unidad Académica Caleta Olivia

Recibido: 06/06/2018. Aceptado: 25/10/2019

\section{RESUMEN}

El crecimiento poblacional y la aparición de nuevas tecnologías (que han mejorado el bienestar humano) (Índice de desarrollo humano, s.f) han producido una mayor demanda energética. Igualmente se ha observado una actitud de "derroche energético" como se los puede observar en el siguiente enlace (Derroche energético: ¿cuál es la situación actual?, s.f). Se estudiará los hábitos energéticos en una casa-tipo (consumo y demanda) con el objetivo de cuantificar la energía necesaria (demanda) y parte de ella obtenerla con recursos no convencionales renovables.

Se procederá a realizar una breve descripción de las fuentes renovables a utilizar (solar, eólica y biomasa) y a través del estudio, que se ha realizado, ver los equipos posibles a instalar en esta casa-tipo. Al mismo tiempo, comprenderemos el porqué del uso de este tipo de tecnologías en locaciones un tanto apartadas de los medios convencionales de energías.

Palabras clave: solar; eólica; biomasa; energía; abastecimiento.

\begin{abstract}
Population growth and the emergence of new technologies (which have improved human well-being) have produced a greater energy demand. Likewise, an attitude of "energy waste" has been observed, as can be observed in the following link.

Energy habits will be studied in a house-type (consumption and demand) with the objective of quantifying the necessary energy (demand) and part of it to obtain it with renewable nonconventional resources.

We will proceed to make a brief description of the renewable sources to be used (solar, wind and biomass) and through the study, which has been carried out, see the possible equipment to be installed in this type house. At the same time, we will understand the reason for the use of this type of technology in locations somewhat away from conventional energy resources.
\end{abstract}

Key words: solar; wind; biomass; energy; supply. 


\section{INTRODUCCIÓN}

Abastecimiento energético: Proceso por el cual un consumidor, empresa, estado, etc., realiza el aprovisionamiento de los recursos energéticos necesarios para el desarrollo de su actividad (Abastecimiento energético, s.f).

Se hace menester la definición de recurso energético para comprender del tema que se está tratando: Se considera recurso energético a toda aquella sustancia de la cual podemos obtener energía a través de diversos procesos. El amplio grupo de sustancias que conforman el conjunto de los recursos energéticos puede ser agrupado en dos categorías generales en función de su proceso de formación y de su disponibilidad (Recurso energético, s.f).

Recursos no renovables: son todos aquellos recursos, cuya tasa de consumo es superior a su tasa de síntesis.

Recursos potencialmente renovables: son todos aquellos recursos en los que su tasa de síntesis es mucho menor que los NO RENOVABLES, pero su velocidad de consumo debe ser delimitada.

Recursos renovables: son todos aquellos en donde se puede obtener energía de manera ilimitada, el origen de los mismos se encuentra en el SOL.

\section{ENERGÍA SOLAR}

Obtenida a partir del aprovechamiento de la radiación electromagnética procedente del Sol. El hombre la ha empleado desde el origen de los tiempos, pero con los grandes avances tecnológicos, y partir del problema de abastecimiento energético, se han ido perfeccionando los sistemas para colectar el calor y la luz del Sol. Se planea hacer uso de la energía solar activa, la cual hace un buen uso mediante la utilización de paneles solares, como los que se describirán en el siguiente trabajo. Para el mismo se ha hecho de información con el fin de conocer los precios y la instalación de los mismos.

En los últimos años se han desarrollado diversas ramas en cada disciplina con el fin de sacarle el mayor provecho a ésta. Incluso la Agencia Internacional de la Energía ha afirmado:

"El desarrollo de tecnologías solares limpias, baratas e inagotables supondrá un enorme beneficio a largo plazo. Aumentará la seguridad energética de los países mediante el uso de una fuente de energía local inagotable y, aún más importante, independientemente de importaciones, aumentará la sostenibilidad, reducirá la contaminación, disminuirá los precios de los combustibles fósiles. Estas ventajas son globales. De esta manera, los costes para su incentivo y desarrollo deben ser considerados inversiones, deben ser realizadas de forma correcta y ampliamente difundidas." (Agencia Internacional de la Energía, 2011)

\section{ENERGÍA EÓLICA}

Energía renovable que cada vez más, se ha ido instalando en nuestro paisaje en forma de aerogeneradores (ubicándose como el $7 \mathrm{mo}$ país en capacidad instalada eólica en Latinoamérica (Argentina se posiciona como el $7^{\circ}$ país en capacidad instalada eólica en Latinoamerica, 2 de marzo de 2018)). En la región de la Patagonia resulta muy conveniente y 
aprovechable debido a los vientos existentes en la zona, dónde existen registros de velocidades elevadas en la estancia que se está estudiando.

Tiene, en realidad, su origen en el Sol, por la diferencia de temperaturas existente en las distintas capas de aire de la atmósfera, produciendo el viento que es captado por los aerogeneradores, que alcanzan vientos a una altura de 50 metros de altura por las hélices de hasta $23 \mathrm{~m}$ de longitud.

El viento mueve las hélices (o palas) y gracias a un rotor de un generador. Convierte la fuerza del viento en energía eléctrica.

Podemos mencionar como ventajas de la energía eólica que no son necesarios complicados procesos de producción y es, totalmente, renovable siendo una alternativa mucho más limpia que el carbón, el petróleo, el gas natural o la energía nuclear. No produce residuos (gases de efecto invernadero, basura, etc.) que puedan afectar al medioambiente.

Entre los inconvenientes encontramos que para funcionar a pleno rendimiento se necesitan ciertas condiciones meteorológicas, de acuerdo al tipo de aerogenerador a instalar. Otro punto es que causa un importante impacto paisajístico en la zona y puede afectar a la vida y costumbres de los animales de la zona, especialmente de las aves.

\subsection{BIOMASA}

Es la cantidad de materia acumulada en un individuo, un nivel trófico, una población o un ecosistema.

La biomasa de la madera, residuos agrícolas y estiércol continúa siendo una fuente principal de energía y materia útiles para el abastecimiento energético en zonas aisladas de fuentes convencionales de energía.

La biomasa podría proporcionar energías sustitutivas a los combustibles fósiles, considerando que no se emplee más biomasa que la producción neta del ecosistema explotado.

Las desventajas en el uso de biomasa se encuentran al usar los cultivos vegetales comestibles para la producción de biocombustibles. En cuanto a su proceso de producción: la incineración de los cultivos puede resultar peligrosa y producir sustancia tóxicas. Es por ello que, se deben utilizar filtros y realizar la combustión a temperaturas mayores a los $900^{\circ} \mathrm{C}$.

Se plantea el uso a partir de un biodigestor, ya instalado en el establo de los animales, el mismo permitirá un ambiente agradable para los animales y en la propia casa-tipo.

\subsection{INVERSORES}

Tienen la función de convertir la energía obtenida a partir de las fuentes renovables (Corriente Continua de muy bajo voltaje) en energía de Corriente Alterna, debido a que los aparatos eléctricos han sido diseñados para trabajar con este tipo de energía.

Se tienen diversos tipos según el origen de la energía obtenida (solar-eólica), pero pueden ser integrados en un sistema de abastecimiento energético en conjunto con las baterías. 


\subsection{BATERIAS}

Cumplen la función de almacenar la energía obtenida durante el ciclo de producción de las energías para su posterior uso cuando sea necesario. Existen diferentes tipos, según sea por la autonomía requerida y/o trabajo de carga/descarga de las mismas.

Al tratarse de un sistema OFF-GRID (desconectado de línea) serán necesarias en gran cantidad para el abastecimiento adecuado de la casa-tipo cumpliendo con todos los requisitos energéticos de la misma.

\section{UBICACIÓN DE LA CASA-TIPO}

En la Figura 2 se visualiza el porcentaje de luz natural que llega a la vivienda con sus aperturas (puertas y ventanas). El mismo fue realizado con el programa Ecotect.

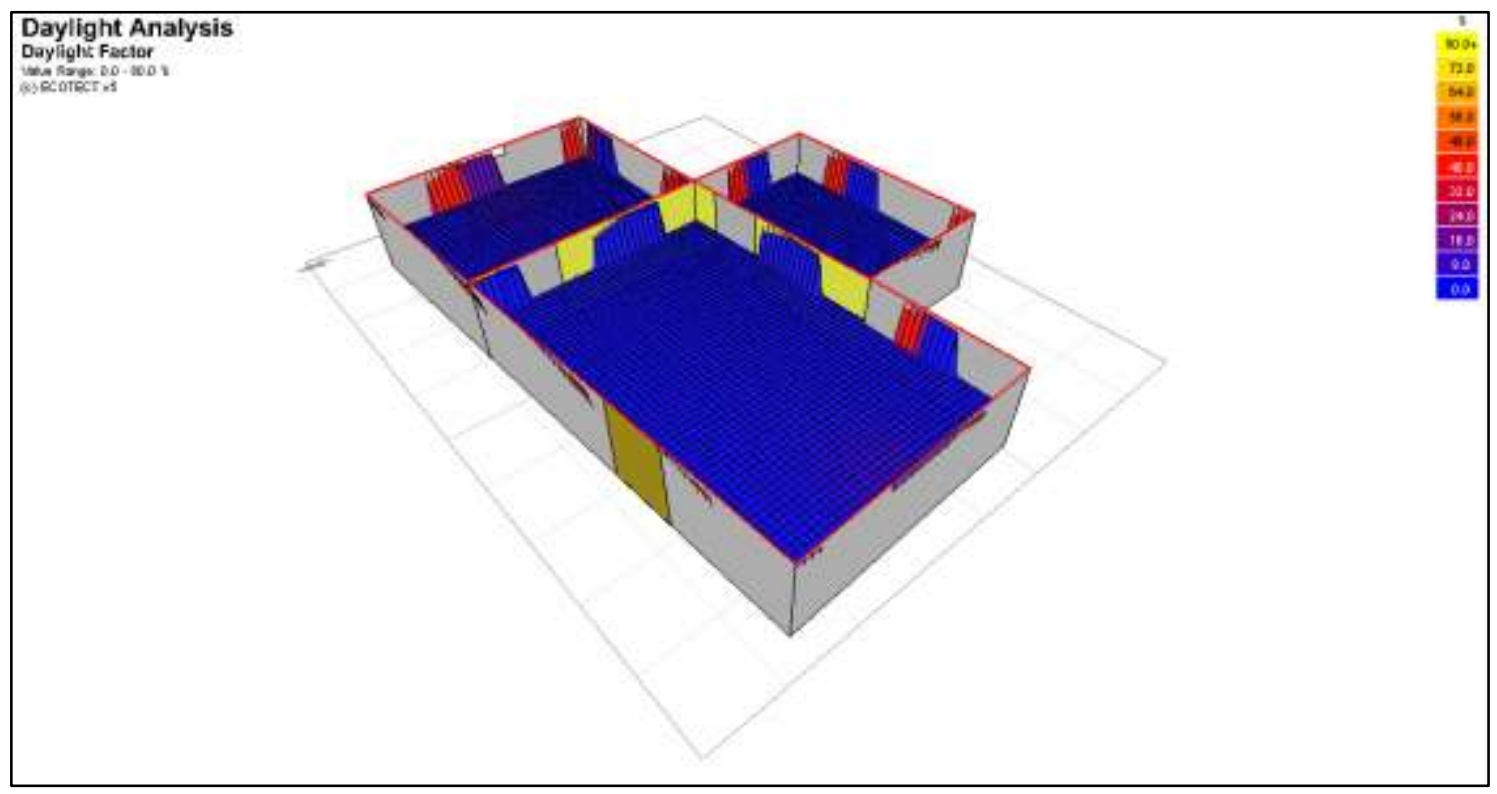

\section{Figura 1. Iluminación natural g}

Se puede observar que los niveles de iluminación son insuficientes para la comodidad de sus habitantes, por ello mismo se realiza el estudio de la cantidad de luz artificial posible de instalar.

En la Figura 3 se observa el porcentaje de luz artificial recibida, realizado con el programa Ecotect. 


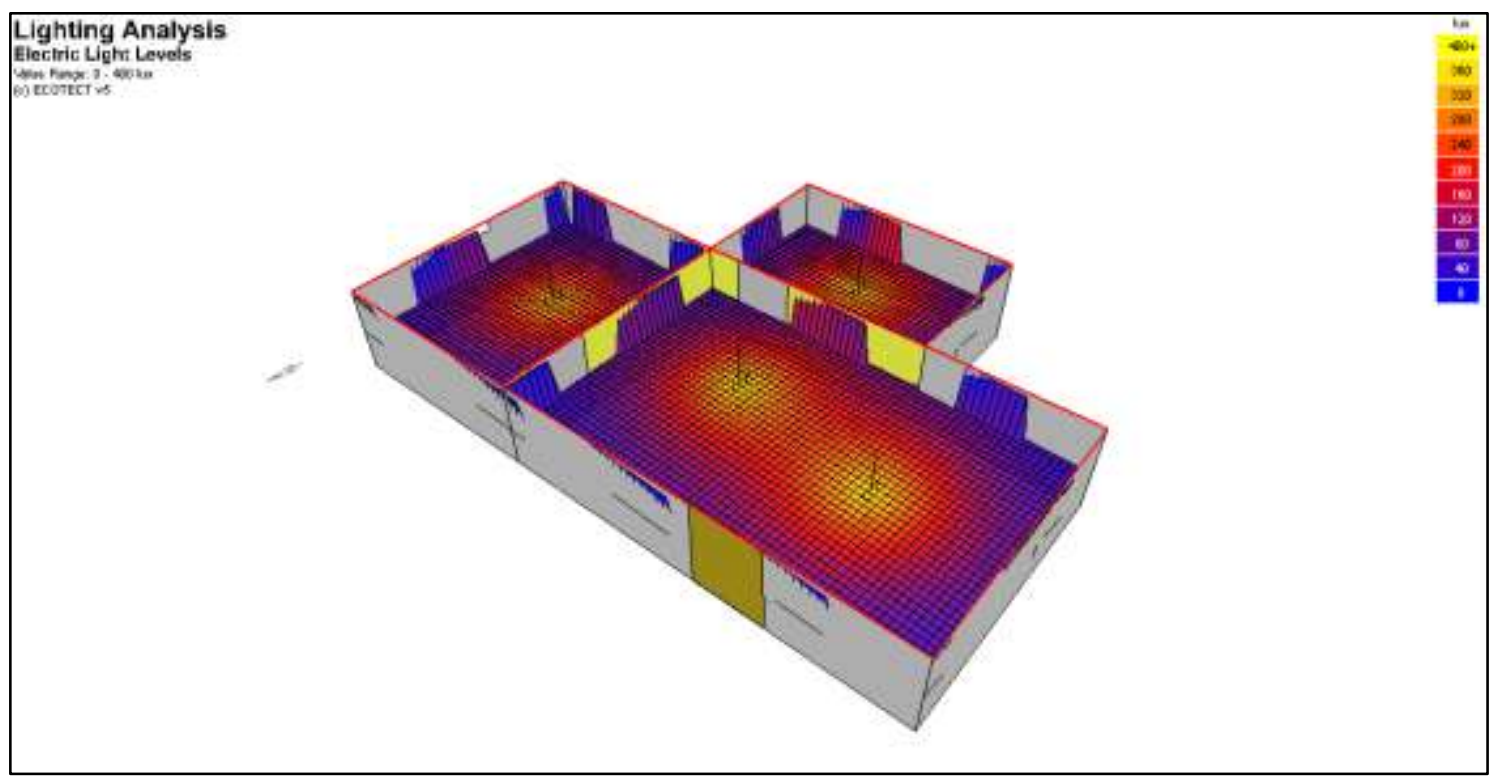

Figura 2. Iluminación artificial

\subsection{CONSUMO PROMEDIO DE LA CASA-TIPO}

Se supone que la casa-tipo y sus alrededores necesitarán energía para los siguientes tipos de elementos e instalaciones:

\section{Casa:}

- Lámparas led de potencia de 10W (vatios) con luminosidad de 774 lumens (Lampara Led 9w Interelec X 50 Foco 220v E27 10w, s.f)

- Biogás para alimentar la habitación mediante Salamandra ${ }^{\mathrm{TM}}$ ya instalada (17 $\mathrm{dm}^{3}$ ) (Salamandras, calefactores o estufas a leña, s.f)

\section{Terreno:}

- Biogás suficiente para alimentar el establo del ganado (ovino y porcino).

La distribución de las lámparas, cantidad y energía consumida por la instalación se detallan en la tabla 1.

\begin{tabular}{|c|c|c|c|c|c|c|c|}
\hline $\mathrm{N}^{\circ}$ & LOCAL & ARTEFACTO & CANTIDAD & $\begin{array}{c}\text { POTENCIA } \\
{[\mathrm{W}]}\end{array}$ & $\begin{array}{l}\text { POTENCIA } \\
\text { TOTAL [W] }\end{array}$ & $\begin{array}{c}\text { HORAS/ } \\
\text { DÍA }\end{array}$ & $\frac{(\text { W.hs }) /}{\text { día }}$ \\
\hline 1 & $\begin{array}{l}\begin{array}{l}\text { Cocina- } \\
\text { comedor }\end{array} \\
\end{array}$ & Lámpara LED & 2 & 10 & 20 & 4 & 80 \\
\hline 2 & Habitación & Lámpara LED & 1 & 10 & 10 & 2 & 20 \\
\hline 3 & Baño & Lámpara LED & 1 & 10 & 10 & 1 & 10 \\
\hline TOTAL & & & & & 40 & & 110 \\
\hline
\end{tabular}

Tabla 1. Consumo energético de la casa 
Como se puede observar en la Imagen 4, mediante la utilización de lámparas LED se evita un consumo de electricidad excesivo, además, se observa que tienen una iluminación similar, reflejada en los lux que emiten. La vida útil de las mismas lámparas es mayor al tratarse de lo que denominan $<<$ luz fría $>>$.

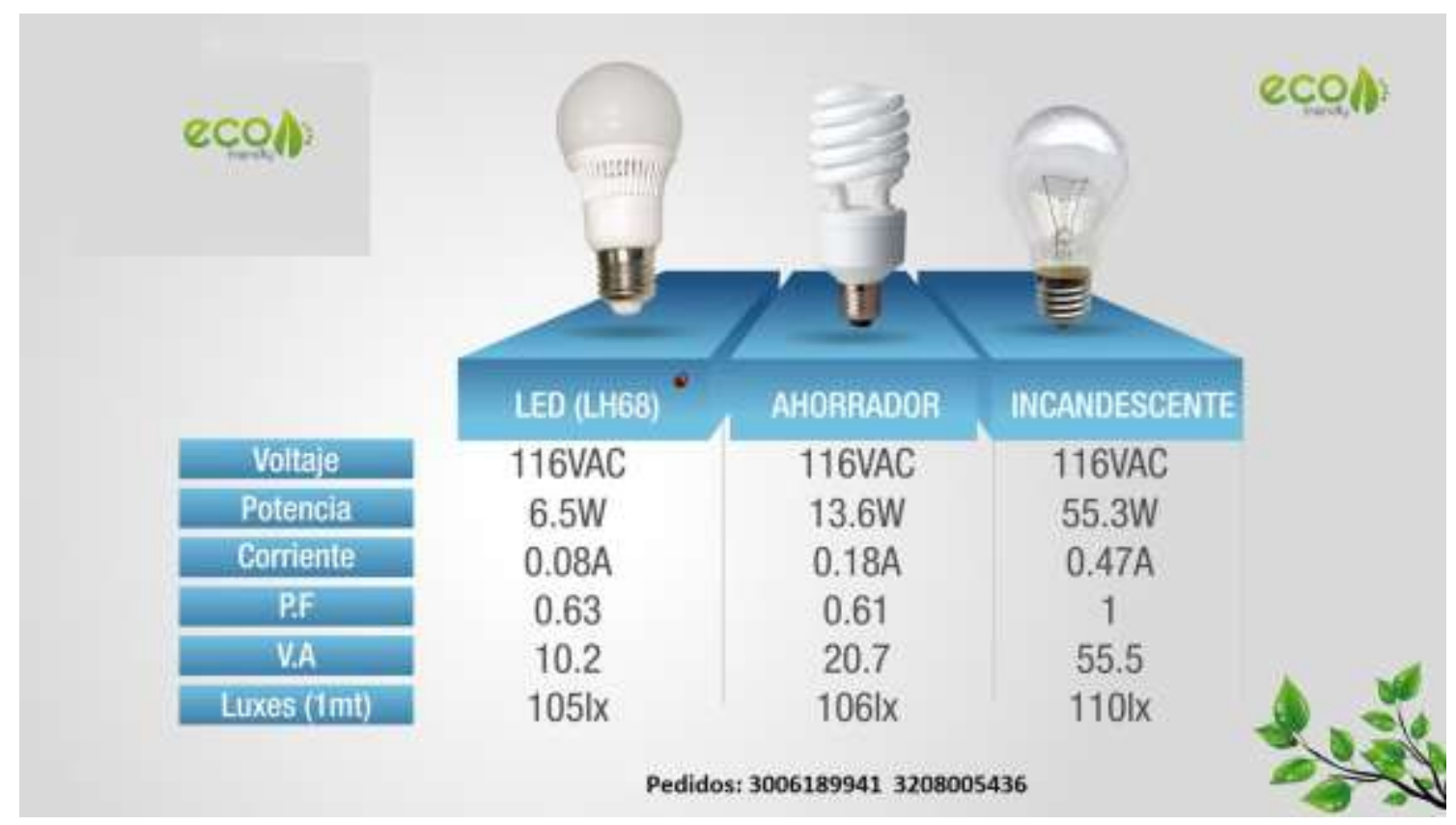

\section{Figura 4. Consumo energético Iluminación}

El consumo promedio de gas por habitante en un hogar argentino es de $1006 \mathrm{~m}^{3} /$ año (La Argentina tiene el consumo más alto de gas por habitante en América del Sur, 16 de agosto de 2016), que es el mayor de América del Sur, ubicándose como el mayor consumo per cápita a nivel mundial.

Al consumo energético de $110 \mathrm{~W}$.hs/día se le considera un incremento del 20\% considerando: caída de tensión, pérdidas en baterías, consumo interno de los elementos a utilizar, momento más desfavorable (invierno), entre otras pérdidas. Así, las potencias serán:

Potencia Instantánea Total $\quad P_{i}=40 \mathrm{~W}+20 \%=48 \mathrm{~W}$

Consumo total del Proyecto $P=110 \frac{W \cdot h s}{d i ́ a}+20 \%=132 \frac{W \cdot h s}{d^{i} a}$

A partir del conocimiento de todas las necesidades energéticas del lugar, y sabiendo de los grandes costos que implicaría la llegada de la energía a través de SPSE (Servicio Públicos Sociedad del Estado) y Distrigas, siendo las empresas proveedoras de energía en la zona, ahora se procederá a la descripción de energías renovables, que si bien implican un costo determinado, debido a las grandes distancias y el aislamiento de la casa-tipo, los mismos resultan factibles y a realizar en un corto-mediano plazo.

Se empezará con la energía solar, luego la eólica y, finalmente, energía a partir de biomasa. 


\section{ENERGÍA SOLAR}

Se hará uso de la energía del Sol, mediante la utilización de paneles fotovoltaicos (correspondiendo a la energía solar activa) ya que como se ha observado, en cuanto a la porción que corresponde a la parte pasiva, no se pueden hacer cambios inmediatos, debido a los grandes costos que implican su implementación.

Medidas del panel solar: largo 0,54m x ancho 0,675m x espesor 0,03m

A partir de las medidas, la superficie del panel aprovechable: $S=0,3645 \mathrm{~m}^{2}$

Ahora, se procede a evaluar la inclinación óptima que deben tener los paneles solares:

$\beta_{\text {opt }}=3,7+0,69 * / \varphi /=35,738977^{\circ}$

Latitud de la ciudad de Caleta Olivia: $-46,4333^{\circ}$

$/ \phi /$ latitud del lugar sin signo (grados)

$\beta$ ángulo de inclinación optima (grados)

A partir de los datos extraídos de la base de datos de la NASA (National Aeronautics and Space Administration) (Tabla 2), se procederá al cálculo del número de paneles necesarios, como así también el número de baterías mediante el uso de FEBO.

\section{Monthly Averaged Insolation Incident On A Horizontal Surface $\left(\mathrm{kWh} / \mathrm{m}^{2} / \mathrm{day}\right)$}

\begin{tabular}{|l|l|l|l|l|l|l|l|l|l|l|l|l|l|}
\hline $\begin{array}{l}\text { Lat } \\
\text { Lon -67.533 }\end{array}$ & Jan & Feb & Mar & Apr & May & Jun & Jul & Aug & Sep & Oct & Nov & Dec \\
\hline 22-year Average & 6.84 & 5.96 & 4.33 & 2.78 & 1.65 & 1.22 & 1.43 & 2.27 & 3.57 & 5.11 & 6.41 & 6.97 \\
\hline
\end{tabular}

\section{Tabla 2. Promedio mensual de insolación sobre una superficie horizontal (NASA}

FEBO (Figura 5) es un programa libre realizado con fines educativos por el Sr. Funes, Federico en el año 2016 como proyecto final en el IPES (Instituto Provincial Educación Superior) de la localidad de Pico Truncado para obtener su título como técnico superior en energía renovables, para el mismo se precisa la información aportada por la NASA (Figura 6) y el mismo nos brindará información acerca del número de paneles fotovoltaicos y baterías para el abastecimiento energético de la misma.

En la Figura 7 se observan los parámetros para los cuales se ha realizado el cálculo de los paneles fotovoltaicos, observando una potencia de panel de $50 \mathrm{~W}$, un factor de seguridad de 1,2 y de 4 días sin generación (un estándar al momento de cálculos fotovoltaicos). En la Figura 8 se observa el consumo energético sobre el cuál se hará el cálculo (anteriormente descripto en la Tabla 1).

En la Figura 9, se evidencian los parámetros de eficiencia del sistema, dónde se tiene en cuenta las eficiencias del regulador, de las baterías y del convertidor, que son las eficiencias que se deben considerar a la hora de obtener el rendimiento total del sistema. Mientras que en la Figura 10 se observan las propiedades de las baterías para tener conocimiento de la energía entregada por cada batería. 


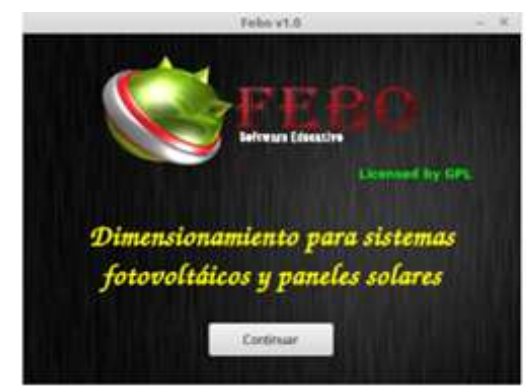

Figuras. FeBo

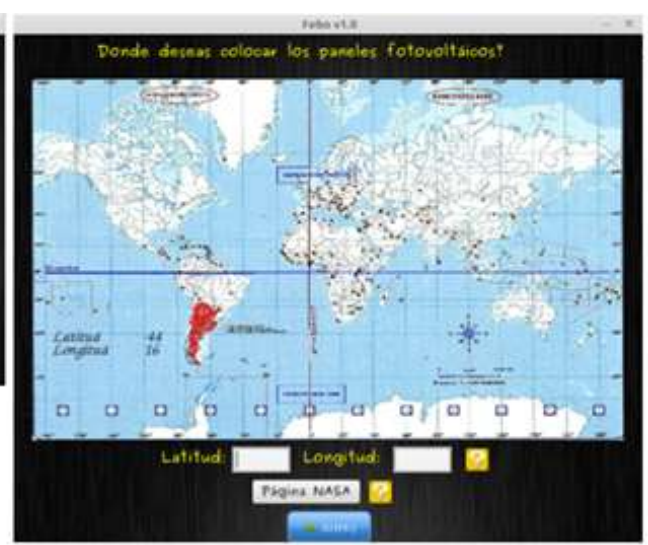

-Figura 6. Vista previa de cómo obtener los datos a partir de la latitud y longitud

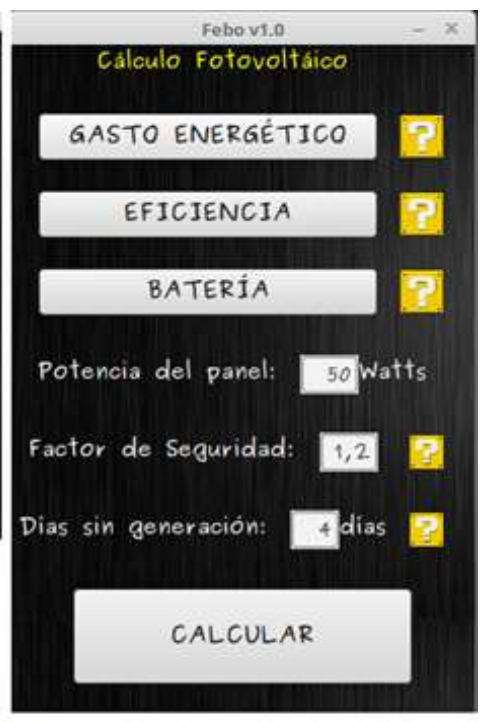

Figura 7. Datos paca el cálculo fotovedtaico

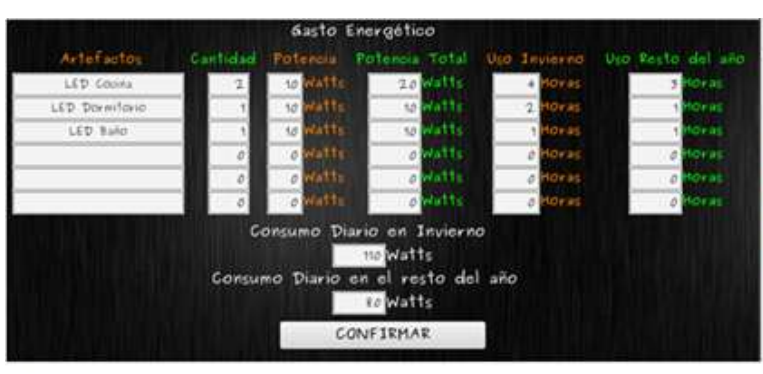

Figurak. Gastoenergético

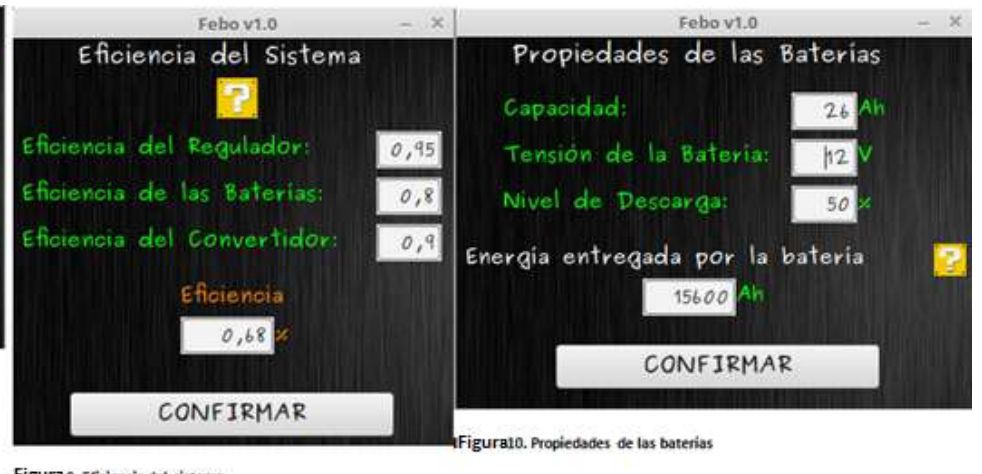

Figura9. Eficiencia del sistems 


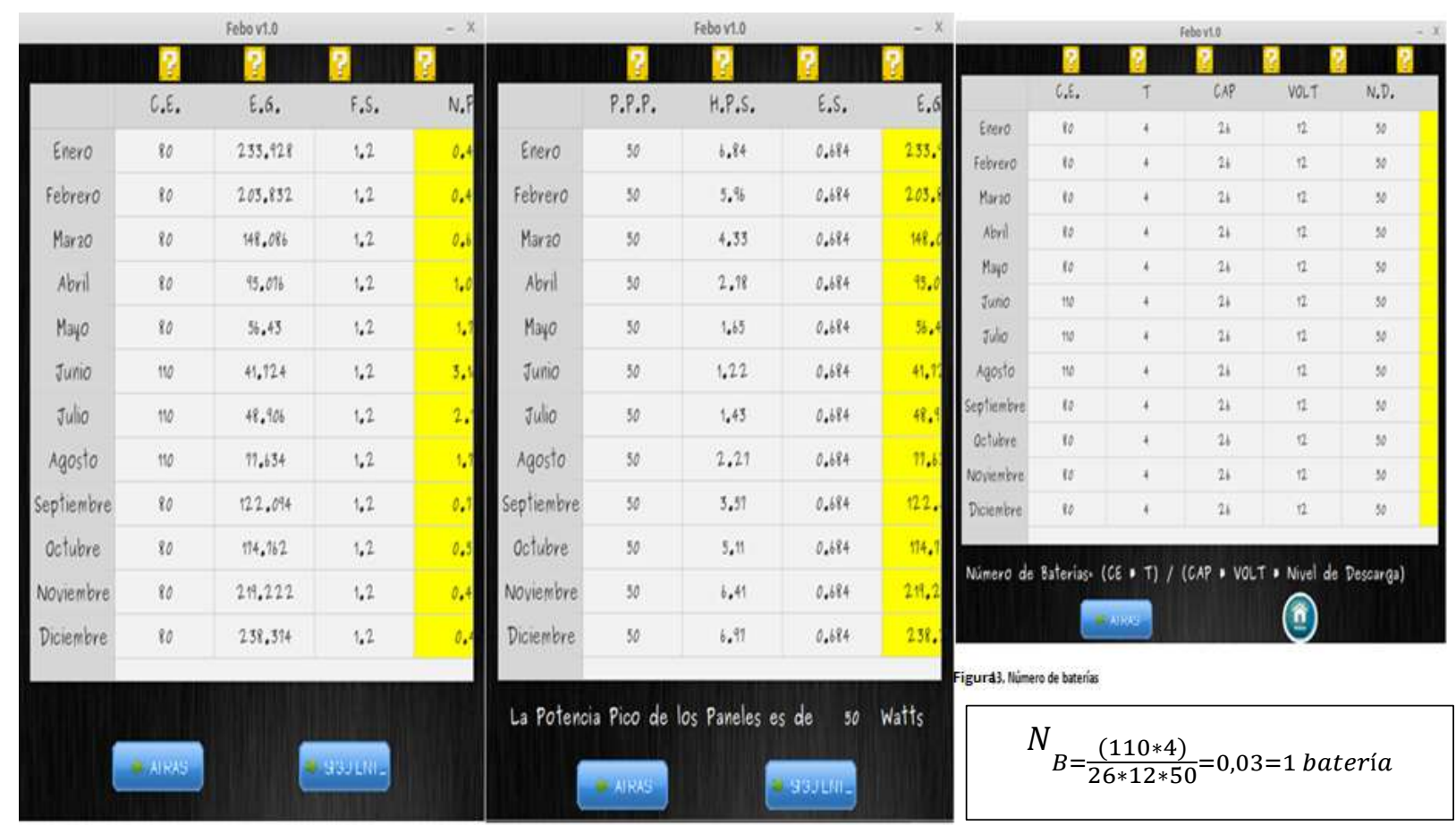

Los cálculos se han realizado a partir del panel fotovoltaico de $50 \mathrm{~W}$, con una batería de 26 $\mathrm{Ah}$, un regulador de carga de $5 \mathrm{~A}$ y un inversor de $150 \mathrm{~W}$, siendo el mismo el abastecido en el kit solar examinado. Todos los artículos están diseñados para funcionar en conjunto de manera adecuada. (Kit Panel Solar 50w Regulador 10a Bat 24amp Inversor 150w, s.f)

El programa nos proporciona información para abastecer energéticamente los elementos descriptos anteriormente, si fuese necesario aumentar el número de aparatos a abastecer, bastaría con agregarlos en el programa para una mayor información.

Ahora, se procederá a la exposición de los resultados arrojados por el software, el mismo nos permitirá una mejor compresión del recurso y, así, comenzar con el presupuesto que demandaría la adquisición de los elementos del sistema fotovoltaico.

La Figura 11, 12 y 13 nos muestran los resultados obtenidos a partir del consumo energético de los elementos de luminaria de la casa tipo.

En primer lugar se dará a conocer el significado de las siglas para un mejor conocimiento de la experiencia:

Figura 11:

- $\quad$ C.E. $=$ Consumo energético

- E.G.= Energía útil generada por cada panel

- F.S.= Factor de seguridad

- N.P.= Número de paneles necesarios 
Figura 12:

- P.P.P.= Potencia pico del panel

- H.P.S.= Horas picos solares

- $\quad$ E.S.= Eficiencia del sistema

- E.G.= Energía útil generada por cada panel

Figura 13:

- C.E. $=$ Consumo energético

- $\mathrm{T}=$ Días sin generación

- $\mathrm{CAP}=$ Capacidad de la batería

- $\quad \mathrm{VOLT}=$ Tensión de la batería

- $\quad \mathrm{ND}=$ Nivel de descarga de la batería

- N.B.= Número de baterías

Se puede observar que el software permite el dimensionamiento de los paneles fotovoltaicos mínimo para cumplir con los requisitos de consumo energético. En caso de incrementar la energía requerida, también será necesario colocar un mayor número de paneles para satisfacerlo.

Estos valores son considerados estándar con paneles fotovoltaicos nuevos y que sea encuentren en condiciones óptimas, para lo cual es imprescindible realizar el mantenimiento de los mismos para que no se vea alterada su capacidad de producción.

"Artículo elaborado por el departamento técnico de SunFields Europe" (Mantenimiento de tu instalación fotovoltaica. Recomendaciones para Propietarios e Instaladores, s.f).

\section{ENERGÍA EÓLICA}

También se trata de hacer uso de la energía a partir del Sol; pero más bien es por las diferencias de presión que se generan en la atmósfera provocando la aparición de vientos sobre el continente (de igual manera sobre los océanos). Los vientos han sido observados durante bastante tiempo, y también han sido utilizados, y la energía eólica nos propone generación de energía a través de los vientos.

La zona sobre la que se está realizando el estudio puede verse favorecida mediante la utilización de aerogeneradores para transformar dicha energía. Se procederá a realizar una descripción del recurso eólico en la zona y buscar las alternativas que serían más acordes para el abastecimiento energético de la casa. Se buscará realizar el análisis sobre el total del consumo energético; pero, lo que se plantea es la diversificación de los recursos energéticos y no la elección de uno de los dos.

La Tabla 3 nos muestra un promedio de 22 (veintidós) años de medidas de la velocidad del viento a diferentes alturas $(50,100,150$ y $300 \mathrm{~m})$ con una vegetación del tipo "hierba áspera plana". 


\begin{tabular}{|c|c|c|c|c|c|c|c|c|c|c|c|c|c|}
\hline \multicolumn{14}{|c|}{$\begin{array}{c}\text { Monthly Averaged Wind Speed At 50, 100, } 150 \text { and } 300 \mathrm{~m} \text { Above The Surface Of The } \\
\text { Earth }(\mathrm{m} / \mathrm{s}) \\
\text { Vegetation type "Airport": flat rough grass }\end{array}$} \\
\hline $\begin{array}{l}\text { Lat }-46.433 \\
\text { Lon }-67.533\end{array}$ & Jan & Feb & Mar & Apr & May & Jun & Jul & Aug & Sep & Oct & Nov & Dec & $\begin{array}{l}\text { Annual } \\
\text { Average }\end{array}$ \\
\hline $50 \mathrm{~m}$ & 8.06 & 7.17 & 6.92 & 7.44 & 7.08 & 6.86 & 7.06 & 6.92 & 7.06 & 7.28 & 7.89 & 8.06 & 7.31 \\
\hline $100 \mathrm{~m}$ & 8.94 & 7.95 & 7.67 & 8.25 & 7.85 & 7.61 & 7.83 & 7.67 & 7.83 & 8.07 & 8.75 & 8.94 & 8.11 \\
\hline $150 \mathrm{~m}$ & 9.50 & 8.45 & 8.15 & 8.77 & 8.34 & 8.08 & 8.32 & 8.15 & 8.32 & 8.58 & 9.30 & 9.50 & 8.62 \\
\hline $300 \mathrm{~m}$ & 10.5 & 9.38 & 9.05 & 9.73 & 9.26 & 8.97 & 9.23 & 9.05 & 9.23 & 9.52 & 10.3 & 10.5 & 9.57 \\
\hline
\end{tabular}

Tabla 3. Velocidad del viento a distintas alturas $[\mathrm{m} / \mathrm{s}]$ (obtenidas de la base de datos de la NASA)

Como bien sabemos, este es el tipo de vegetación en inmediaciones de la estancia, promedio de la velocidad del viento es de 7,31 m/s a una altura de $50 \mathrm{~m}$.

La velocidad promedio no es de suma importancia para la elección del aerogenerador óptimo a instalar, ya que esté nos indica que el aerogenerador a instalar óptimo sería un tripala de eje horizontal. Sin embargo, la experiencia y la observación de los tipos de vientos en el lugar nos aconsejan que no sea preferente la instalación de los mismos debido a la existencia de ráfagas de viento que superan los $80 \mathrm{~km} / \mathrm{h}$. Con este tipo de velocidades del viento la instalación de un aerogenerador de eje horizontal no es recomendable debido a que sería necesario realizar un mantenimiento excesivo del aerogenerador.

Además, el costo de instalación de uno de tipo de eje horizontal es alto debido a la instalación de la torre sobre que se colocará el aerogenerador. Es por ello, que se recomienda la instalación de un aerogenerador de eje vertical.

La firma"AEOLOS Wind Turbine" ofrece los dos tipos de aerogeneradores para la misma potencia a instalar de $1 \mathrm{~kW}$ de potencia. 


\begin{tabular}{|c|c|}
\hline $\begin{array}{l}\text { Potencia } \\
\text { Nominal }\end{array}$ & $1 \mathrm{kw}$ \\
\hline $\begin{array}{l}\text { Máxima Potencia } \\
\text { de Salida }\end{array}$ & $1.2 \mathrm{kw}$ \\
\hline Voltaje de Salida & $48 \mathrm{~V}$ \\
\hline Número de Palas & $\begin{array}{c}3 \text { Palas de Fibra } \\
\text { de Vidrio }\end{array}$ \\
\hline $\begin{array}{l}\text { Diámetro del } \\
\text { Rotor de Palas }\end{array}$ & $3.2 \mathrm{~m}$ (10.5 pies) \\
\hline $\begin{array}{c}\text { Velocidad de } \\
\text { Arranque del } \\
\text { Viento }\end{array}$ & $\begin{array}{c}2.5 \mathrm{~m} / \mathrm{s}(5.6 \\
\mathrm{mph})\end{array}$ \\
\hline $\begin{array}{c}\text { Velocidad } \\
\text { Nominal del } \\
\text { Viento }\end{array}$ & $\begin{array}{c}12 \mathrm{~m} / \mathrm{s}(26.8 \\
\mathrm{mph})\end{array}$ \\
\hline $\begin{array}{l}\text { Velocidad de } \\
\text { Sobrevivencia } \\
\text { del Viento }\end{array}$ & $\begin{array}{c}45 \mathrm{~m} / \mathrm{s}(100.7 \\
\mathrm{mph})\end{array}$ \\
\hline Generador & $\begin{array}{c}\text { Generador } \\
\text { Trifásico } \\
\text { Magnético } \\
\text { Permanente }\end{array}$ \\
\hline $\begin{array}{c}\text { Eficiencia del } \\
\text { Generador }\end{array}$ & $>0.96$ \\
\hline Peso de Turbina & $60 \mathrm{~kg}$ (132.3 lbs) \\
\hline Ruido & $30 \mathrm{db}(\mathrm{A}) @ 5 \mathrm{~m} / \mathrm{s}$ \\
\hline $\begin{array}{c}\text { Rango de } \\
\text { Temperatura }\end{array}$ & $-20^{\circ} \mathrm{C} \mathrm{a}+50^{\circ} \mathrm{C}$ \\
\hline $\begin{array}{l}\text { Vida Útil del } \\
\text { Diseño }\end{array}$ & 20 Años \\
\hline Garantía & Estándar 5 Años \\
\hline
\end{tabular}

Tabla 4. Especificaciones técnicas aerogenerador eje horizontal (Aerogenerador 1kw, s.f)

\begin{tabular}{|c|c|}
\hline $\begin{array}{c}\text { Potencia } \\
\text { Nominal }\end{array}$ & $1 \mathrm{kw}$ \\
\hline $\begin{array}{c}\text { Máxima Potencia } \\
\text { de Salida }\end{array}$ & $1500 \mathrm{w}$ \\
\hline Voltaje de Salida & $48 / 110 \mathrm{~V}$ \\
\hline $\begin{array}{c}\text { Altura del Rotor } \\
\text { Diámetro del } \\
\text { Rotor }\end{array}$ & $2.8 \mathrm{~m}(9.2$ pies $)$ \\
\hline $\begin{array}{c}\text { Velocidad de } \\
\text { Arranque del } \\
\text { Viento }\end{array}$ & $1.5 \mathrm{~m} / \mathrm{s}(3.4$ \\
$\mathrm{mph})$
\end{tabular}

Tabla 5. Especificaciones técnicas aerogenerador eje vertical (Aerogenerador Vertical Aeolos-V $1 \mathrm{kw}, \mathrm{s.f})$ 
Teniendo las especificaciones de ambos modelos, se realiza la comparación entre los dos y no se observa gran diferencia entre lo que cada uno de ellos aporta. Se puede realizar la instalación de cualquiera de ellos; pero, teniendo en cuenta el aumento económico que supondría el de eje horizontal, se optará por el de eje vertical.

Ventajas de los aerogeneradores de eje vertical (Beneficios de los aerogeneradores de eje vertical, s.f)

- No requieren grandes inversiones porque sus dimensiones pueden ser reducidas. Tampoco necesitan de una torre potente o instalaciones complejas como los de eje horizontal. Su mantenimiento es sencillo, y se pueden colocar en cualquier lugar, tanto sobre suelo firme o sobre el tejado de una vivienda.

- Aprovechan mejor la dirección de los vientos, no importa si son turbulentos o de baja altura, tampoco afecta las irregularidades del terreno, que incrementan la velocidad del viento. La turbina vertical aprovecha el mínimo flujo de aire para obtener energía limpia.

- Funcionan de manera silenciosa, algo fundamental para que puedan ubicarse en lugares habitados, tanto urbanos como rurales. Tienen la ventaja de que se ponen en marcha con pequeñas velocidades del viento, a la vez que son más resistentes a las ventoleras intensas.

- Su impacto ambiental es mucho menor, ya que las aves pueden evitarlos con mayor facilidad y no es imprescindible que estén en espacios naturales para aprovechar la energía eólica.

- Su alineación vertical recibe al viento desde cualquier dirección, por lo que no requieren ningún sistema de alineación del aerogenerador, lo que reduce significativamente su coste. Esta virtud también es muy útil en lugares donde el viento cambia de forma rápida, como suele ser en medio del mar.

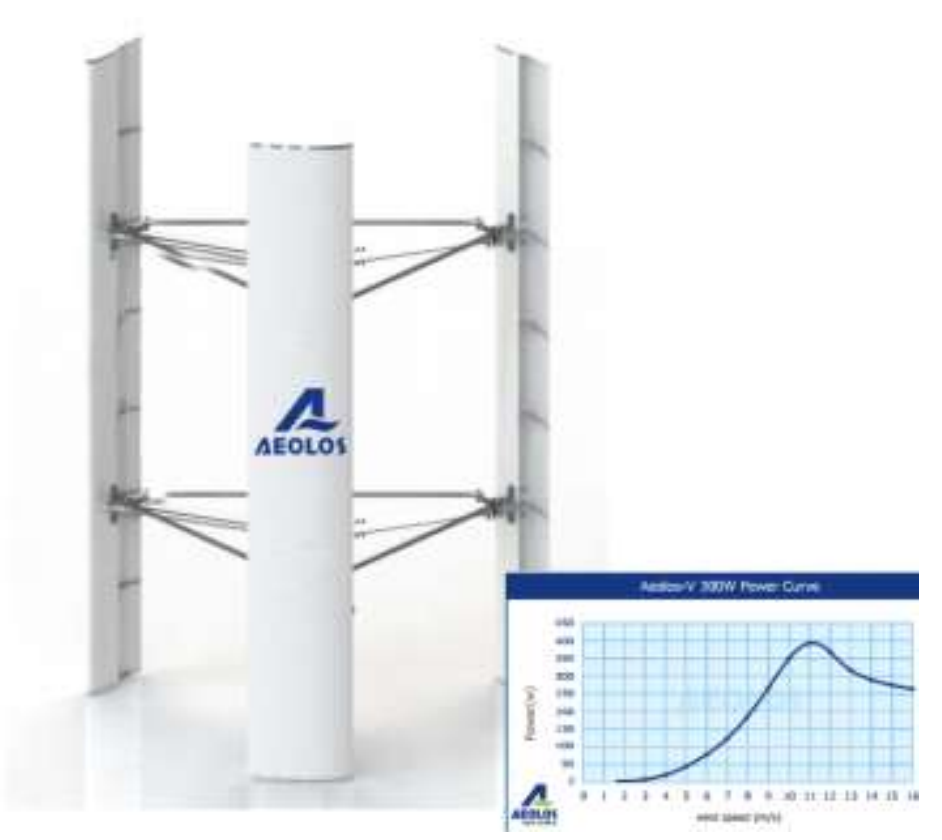

Figura 15, Aerogenerador de deje vertical junto con su curva de potencia 
En la imagen 14, logramos apreciar el aerogenerador de eje vertical que se procederá a instalar y a su lado se encuentra la curva de potencia del mismo. En la misma, es posible apreciar que el aerogenerador puede producir energía en un gran rango de velocidades; el máximo rendimiento se encuentra cuando velocidad del viento es de $11 \mathrm{~m} / \mathrm{s}$, pero el rango de velocidades en las que sería posible la generación es mucho mayor.

A partir de la misma, se llega a la conclusión de que la instalación de este tipo de aerogenerador en conjunto con el sistema de paneles solares será de un gran beneficio para la estancia hablando desde el abastecimiento energético. Además, se ha conocido que el mantenimiento que requiere es mucho menor que los aerogeneradores de eje horizontal, presuponiendo un mantenimiento menos intenso y un mejor rendimiento general por parte del mismo.

\section{BIOMASA}

Se procederá a la descripción de abastecimiento energético de gas del lugar mediante la utilización de material propio de la labor de la estancia, como lo es el estiércol que producen los animales.

Éste permitirá, mediante la utilización de un biodigestor, el abastecimiento del recurso para calefacción de la residencia y del establo en donde se encuentra el ganado.

La estancia cuenta con:

- 200 ovejas

- 100 cerdos

Es a partir de éstos que se realizarán cálculos para conocer el volumen de biogás factible de generar en plaza. La calefacción de los lugares está pensada realizarse mediante el uso de estufas Salamandras TM, que permiten la calefacción del lugar utilizando gas sin importar su origen. En general, las mismas utilizan gas obtenido a partir de hidrocarburos; pero, se puede hacer uso de las mismas a partir del poder calorífico del biogás.

El objetivo es el de enseñar los beneficios que tienen el aprovechamiento de los materiales considerados desechos, de esta manera se observa la variabilidad de recursos existentes para abastecer energéticamente un lugar.

Se procederá con la descripción de cómo realizar un biodigestor casero para obtener biogás con el fin de lograr su quema para calefacción del lugar deseado.

MATERIALES Y DESCRIPCIÓN (Cómo hacer un biodigestor casero?, 23 de febrero de 2019)

\section{EL REACTOR Y LA ENTRADA DE MATERIALES.}

Un tanque o bidón de entre 120 y 220 litros de capacidad. Generalmente son azules con tapa de cierre hermético.

Tapón de limpieza sanitario (4”): Es una especie de adaptador con tapón enroscable 
Segmento corto de tubo (4"): Pasa a través de la abertura y conecta el "adaptado-tapón" en el exterior con la Reducción en la parte interna del tanque. Debe ser suficientemente corto para permitir que tanto la Reducción como el adaptador-tapón aprisionen la pared de la tapa del tanque y así permitir una mejor sujeción y sellamiento. También se pueden usar bridas sanitarias pegadas con silicona al tanque.

Reducción PVC de 4" a 3"

Tubo PVC sanitario (3”): Desde la reducción hasta 5cm antes del fondo del tanque.

\section{PARA LA SALIDA DEL EFLUENTE:}

Adaptador de tanque (2”).

Tubo PVC (2") para la tubería de salida del efluente.

3 Codos PVC (2”).

Adaptador de tanque (1”) para conectar la válvula.

Válvula de esfera PVC (1”) Para la salida inferior del efluente más pesado.

\section{PARA LA SALIDA DEL BIOGÁS (EN ORDEN):}

Conector de tanque (1/2").

Válvula de esfera con roscas (1/2”).

Adaptador para manguera.

Manguera.

\section{PARA UNIR LAS PARTES Y SELLAR:}

Soldadura (pegamento) para PVC.

Silicona selladora transparente, ¡resistente a hongos!: Para sellar alrededor de las uniones al tanque e impedir filtración.

$\mathrm{Al}$ tanque se le realizan dos agujeros laterales y dos en la tapa. Uno en la parte lateral-inferior para la válvula de 1 pulgada; otro en la parte media para la salida de efluente. En la tapa uno será para la entrada del material y el otro para la salida del biogás, siempre del diámetro de la pieza que lo atraviesa.

PARA ALMACENAR EL BIOGÁS se utiliza un depósito de campana flotante, muy fácil de construir con dos bidones; uno grande donde va el agua y otro ligeramente más angosto que se sitúa boca abajo dentro del anterior. La manguera que viene del digestor se introduce al tanque mayor y burbujea de tal forma que el gas sube y queda atrapado en el tanque menor el cual tiene una válvula para la salida del gas con una manguera y una trampa de agua.

Para poder utilizar el biodigestor su constructor deberá instalar previamente las conexiones, mangueras, válvula de seguridad, depósito de biogás y quemador, así como también revisar 
las conexiones con el fin de evitar fugas de gas o la entrada de aire al aparato. Ya resueltos estos preparativos se podrá proseguir con el llenado de este.

\section{CARGA}

La carga se constituirá por la mezcla de un 20 a $25 \%$ de material orgánico y de un 80 a $75 \%$ de agua. Parte de este agua puede reemplazarse por el líquido (efluente) tratado que sale del biodigestor también conocida como biol, y de esa forma producir más biogás a expensas de obtener menos fertilizante.

El tiempo de retención vendrá determinado por la temperatura a la que será expuesto el biodigestor (Figura 14).

\begin{tabular}{|l|c|c|}
\hline Región característica & Temperatura $\left({ }^{\circ} \mathrm{C}\right)$ & $\begin{array}{c}\text { Tiempo de retención } \\
\text { (días) }\end{array}$ \\
\hline Trópico & 30 & 20 \\
\hline Valle & 20 & 30 \\
\hline Altiplano & 10 & 60 \\
\hline
\end{tabular}

Figura 16. Tiempo de retención según temperatura

Monthly Averaged Air Temperature At 10 m Above The Surface Of The Earth $\left({ }^{\circ} \mathbf{C}\right)$

\begin{tabular}{|l|l|l|l|l|l|l|l|l|l|l|l|l|l|}
\hline $\begin{array}{l}\text { Lat -46.433 } \\
\text { Lon-67.533 }\end{array}$ & Jan & Feb & Mar & Apr & May & Jun & Jul & Aug & Sep & Oct & Nov & Dec & $\begin{array}{l}\text { Annual } \\
\text { Average }\end{array}$ \\
\hline $\begin{array}{l}\text { 22-year } \\
\text { Average }\end{array}$ & 17.6 & 16.9 & 14.1 & 9.82 & 5.75 & 2.73 & 2.40 & 4.30 & 6.98 & 10.8 & 14.3 & 16.4 & 10.1 \\
\hline Minimum & 12.6 & 12.0 & 9.61 & 5.81 & 2.72 & 0.36 & -0.19 & 1.08 & 2.76 & 6.03 & 9.01 & 11.1 & 6.05 \\
\hline Maximum & 22.4 & 21.7 & 18.4 & 13.8 & 8.86 & 5.26 & 5.18 & 7.81 & 11.4 & 15.4 & 19.4 & 21.2 & 14.2 \\
\hline
\end{tabular}

Tabla 6. Temperatura promedio del aire a $10 \mathrm{~m}$ de altura del nivel del suelo

La Tabla 6 nos muestra que la temperatura a $10 \mathrm{~m}$ de altura sobre el nivel del suelo corresponde al "Altiplano". Sin embargo, consideramos que la temperatura a la cual trabajará el biodigestor a nivel del suelo corresponde a $\operatorname{los} 25^{\circ} \mathrm{C}$.

Esta temperatura marcada es la que permitirá el trabajo del biodigestor. Es por ello que para evitar inconvenientes, el mismo se encontrará bajo techo en el granero dónde encuentran ovejas y chanchos. 
RESULTADOS (Curso de Biomasa y Biocombustible del MSc Labriola, Carlos V. M.)

\begin{tabular}{|c|c|c|c|c|c|r|}
\hline Animal & $\begin{array}{c}\text { Estiércol } \\
\text { húmedo } \\
{[\mathrm{kg} / \text { día }]}\end{array}$ & $\begin{array}{c}\text { Biogás } \\
{[\mathrm{m} 3 / \text { día }]}\end{array}$ & $\begin{array}{c}\text { Solidos } \\
\text { Totales } \\
{[\%]}\end{array}$ & $\begin{array}{c}\text { Solidos } \\
\text { volátiles } \\
{[\% \mathrm{VS}]}\end{array}$ & Número & Estiércol \\
\hline Cerdo & 5 & $\begin{array}{c}0,28 \mathrm{a} \\
0,34\end{array}$ & 25 & 80 & 100 & 500 \\
\hline Ovejas & 1,5 & 0,075 & 32 a 45 & 77 & 200 & 300 \\
\hline Humano & $\mathbf{0 , 2}$ & $\begin{array}{c}0,0110 \mathrm{a} \\
0,0014\end{array}$ & $\mathbf{1 5} \mathbf{a} \mathbf{2 0}$ & $\mathbf{9 0}$ & & 1 \\
\hline
\end{tabular}

Tabla 7. Propiedades del estiércol a presión atmosférica

\begin{tabular}{|c|c|c|c|c|}
\hline & \multicolumn{2}{|c|}{ Solidos volátiles } & \multicolumn{2}{c|}{ COD } \\
\hline $\begin{array}{c}\text { Temp. } \\
{\left[{ }^{\circ} \mathrm{C}\right]}\end{array}$ & $\begin{array}{c}\text { Const. De } \\
\text { producción } C \\
{\left[\mathrm{~m}^{3} /(\mathrm{kg} . \mathrm{VS})\right]}\end{array}$ & $\begin{array}{c}\text { Const. } \\
\text { Cinética } k \\
{[1 / \text { días }]}\end{array}$ & $\begin{array}{c}\text { Const. De } \\
\text { producción } C \\
{\left[\mathrm{~m}^{3} /(\mathrm{kg} . C O D)\right]}\end{array}$ & $\begin{array}{c}\text { Const. } \\
\text { Cinética } k \\
{[1 / \text { días }]}\end{array}$ \\
\hline 34 & 0,402 & 0,083 & 0,347 & 0.081 \\
\hline $\mathbf{2 5}$ & $\mathbf{0 , 2 8 9}$ & $\mathbf{0 , 0 6 9}$ & 0,237 & 0.078 \\
\hline 16 & 0,178 & & 0,164 & 0.026 \\
\hline
\end{tabular}

Constante de corrección

\begin{tabular}{|c|r|}
\hline Cerdo & 0,02 \\
\hline Ovejas & 0,02 \\
\hline Humano & 0,03 \\
\hline
\end{tabular}

Tabla 8. Constantes cinéticas de estiércol de vaca en plantas continuas

A la tabla 8 de constantes cinéticas es menester la aplicación de la constante de corrección para obtener de esta manera un cálculo más preciso sobre el biogás posible de obtener.

Los residuos por día se obtienen al multiplicar el número de individuos por los residuos que produce cada uno (Tabla 9).

\begin{tabular}{|c|r|}
\hline ST Cerdo & 125 \\
\hline ST ovejas & 115,5 \\
\hline ST Humano & 0,035 \\
\hline
\end{tabular}

Tabla 9. Sólido s totales [kg/día] 
Lo necesario a ingresar en el digestor es materia orgánica húmeda con $20 \%$ de materia seca en agua, por lo tanto (Tabla 10)

\begin{tabular}{|c|r|}
\hline Cerdo & 625 \\
\hline Ovejas & 577,5 \\
\hline Humano & 0,175 \\
\hline
\end{tabular}

\section{Tabla 10. Volumen diario por $\mathrm{m}^{3}$ de materia orgánica}

Con un tiempo de retención $\mathrm{R}$ de 45 días, el volumen total V de materia orgánica será:

V=R.v
\begin{tabular}{|c|r|}
\hline Cerdo & 28125 \\
\hline Ovejas & 25987,5 \\
\hline Humano & 7,875 \\
\hline
\end{tabular}

\section{Tabla 11. Volumen total de materia orgánica $\left[\mathrm{m}^{3}\right]$}

La cantidad de $\mathrm{kgVS}$ por $\mathrm{m}^{3}$ por día será:

\begin{tabular}{|c|r|}
\hline Cerdo & 100 \\
\hline Ovejas & 88,935 \\
\hline Humano & 0,0315 \\
\hline
\end{tabular}

\section{Tabla 12. Cantidad de kgVS/día}

De la Tabla 7 extraemos el porcentaje de VS, siendo así (Tabla 13):

$\mathrm{r}=$
\begin{tabular}{|c|r|}
\hline Cerdo & 0,00355556 \\
\hline Ovejas & 0,00342222 \\
\hline Humano & 0,004 \\
\hline
\end{tabular}

\section{Tabla 13. Carga diaria}

De la tabla 8 se obtiene los valores de C (constante de producción) y k (constante cinética de primer orden), y se los multiplica por una constante de corrección $(0,02$ y 0,03$)$ para pasar los valores de constantes cinéticas de estiércol de vaca en plantas continuas a constantes cinéticas de estiércol en plantas continuas. La temperatura que se opto es de 25 grados, que es el valor más cercano a la temperatura promedio de trabajo en el lugar.

K
\begin{tabular}{|c|r|}
\hline Cerdo & 0,00138 \\
\hline Ovejas & 0,00138 \\
\hline Humano & 0,00207 \\
\hline
\end{tabular}

C
\begin{tabular}{|c|r|}
\hline Cerdo & 0,00578 \\
\hline Ovejas & 0,00578 \\
\hline Humano & 0,00867 \\
\hline
\end{tabular}

Tabla 14. Valor de $\mathrm{k}$ y C

Considerando que la concentración de materia digestible inicial $S_{0}=r$. $R$, hallamos $g$, que es el volumen diario de gas (Tabla 15): 


G
\begin{tabular}{|c|r|}
\hline Cerdo & 0,03379512 \\
\hline Ovejas & 0,03005569 \\
\hline Humano & $2,3272 \mathrm{E}-05$ \\
\hline
\end{tabular}

Tabla 15. Volumen diario de gas

El volumen total del gas $\mathrm{G}$ producido será:

$$
G=g \cdot R
$$

$\mathrm{G}$
\begin{tabular}{|c|r|}
\hline Cerdo & 1,52078053 \\
\hline Ovejas & 1,35250616 \\
\hline Humano & 0,00104724 \\
\hline
\end{tabular}

Tabla 16. Volumen total producido $\left[\mathrm{m}^{3}\right]$

Este es biogás que se genera en un tiempo de retención $\mathrm{R}$ de 45 días.

El mismo deberá de acumularse en tiempos en donde no sea necesario su uso para los momentos en los que sí sea necesario su uso para calefacción del hogar y establo en otoño e invierno, momentos durante los cuales las temperaturas exteriores son bajas para continuar con la producción del biogás.

\section{PROYECTO DE INVERSIÓN}

Nota: los precios reflejados han sido obtenidos vía Internet en la web de mercadolibre.com.ar los mismos han sido extraídos con el fin de poder obtener valores de referencia en el mercado. Los mismos pueden verse modificados según la disposición del vendedor (Mercadolibre, s.f.).

Se plantea realizar el plan de inversión necesario para abastecer una residencia ubicada a 7 $\mathrm{km}$ al norte de la ciudad de Caleta Olivia mediante el uso de energías renovables ya que la inversión que debería realizarse en el caso de optar por energías convencionales distribuidas por las empresa SPSE (Servicios Públicos Sociedad del Estado) y Distrigas sería muy elevada.

Es por ello que se hará el estudio de la inversión a realizar para abastecer la casa mediante energía solar, energía eólica y energía obtenida a partir de la biomasa (biogás).

Se plantea un diagrama de obtención diaria de todos los tipos de energías durante el año, teniendo en cuenta que la energía solar bastará para la satisfacción del consumo energético durante el verano y la energía eólica servirá como complemento para completar el resto de energía requerida a lo largo del año. En cuanto al biogás se piensa en comenzar con la producción en los meses de verano (donde la temperatura exterior es la adecuada para continuar la producción del biogás) y utilizarla sobre todo en los meses de invierno para calefacción del hábitat de la casa y del granero en donde se encuentran los animales.

El aerogenerador que se tiene pensado instalar es muy superior al requisito energético, de esta manera se está pensando en artefactos que mejoren el bienestar humano dentro de la casa-tipo. 
Análisis FODA (Fortalezas-Oportunidades-Debilidades-Amenazas)

\begin{tabular}{|c|c|}
\hline Fortalezas & $\begin{array}{l}\text { - Posibilidad de autoabastecimiento energético de la } \\
\text { estancia } \\
\text { - Aprovechamiento de la mayor cantidad de recursos } \\
\text { - Costos bajos en comparación con el uso de recursos } \\
\text { energéticos convencionales } \\
\text { - Mantenimiento escaso a realizar } \\
\text { - Terreno extenso } \\
\text { - Vivienda de } 50 \mathrm{~m}^{2} \text { permitiendo el uso eficiente } \\
\text { - } \text { energético } \\
\text { - Granero ya construido para la cría del ganado } \\
\text { - Mayor aprovechamiento de los recursos energéticos }\end{array}$ \\
\hline Oportunidades & $\begin{array}{l}\text { - Posibilidad de incrementar la producción de biogás } \\
\text { mediante la incorporación de un mayor número de } \\
\text { animales } \\
\text { - Posibilidad de incorporar un mayor número de } \\
\text { electrodomésticos con el fin de mejorar la calidad de } \\
\text { vida } \\
\text { - Posibilidad de incrementar el número de paneles y/o } \\
\text { aerogeneradores }\end{array}$ \\
\hline Debilidades & $\begin{array}{l}\text { - } \begin{array}{l}\text { Necesidad de realizar el mantenimiento } \\
\text { obligatoriamente }\end{array} \\
\end{array}$ \\
\hline Amenazas & $\begin{array}{l}\text { - Cambios radicales de las condiciones meteorológicas } \\
\text { desfavorables para la generación de energía }\end{array}$ \\
\hline
\end{tabular}

\section{Tabla 17. Análisis FODA}

El número de fortalezas y oportunidades es mayor al de las debilidades y amenazas, por lo que se puede concluir que realizar una inversión de este tipo resultaría favorable para dar solución al abastecimiento energético de la casa-tipo. Además, este tipo de tecnologías se encuentran en constante desarrollo, permitiendo una manera de abastecimiento adecuado (según las necesidades a cumplir).

\section{INSTALACIÓN DEL SISTEMA HÍBRIDO EÓLICO-SOLAR}

A continuación se presenta el tipo de instalación eléctrica a realizar en la estancia para que pueda cumplir su objetivo de ser una vivienda autosustentable a partir del recurso eólico-solar del sitio estudiado. 


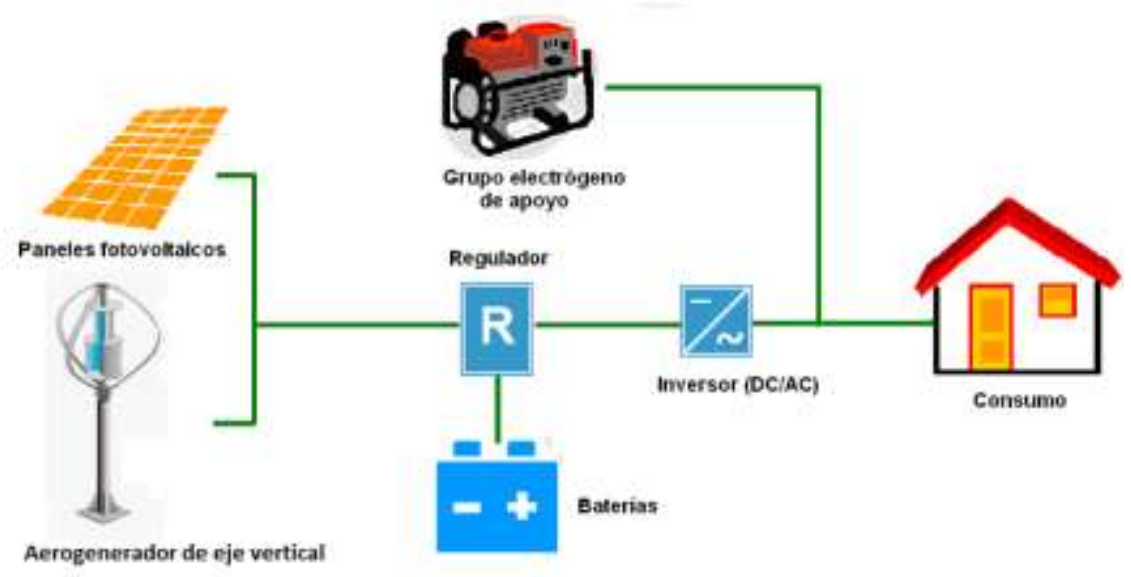

\section{Figura 17. Instalación eléctrica a realizar en la estancia}

Aquí vemos un modelo del tipo de instalación a realizar en la casa-tipo con el fin de cumplir con el requisito fundamental de los productos a adquirir y de la financiación de los mismos, descriptos en el anexo I.

\section{CONCLUSIÓN Y RECOMENDACIONES}

Se ha realizado el estudio de abastecimiento energético de una casa-tipo instalada en la estancia Novosel al norte de la ciudad de Caleta Olivia. La misma no cuenta con los servicios de electricidad y gas natural debido a la distancia que existe del centro urbano de la ciudad de Caleta Olivia.

Esta situación ha permitido el análisis de cómo es posible abastecer energéticamente una residencia mediante el uso de Energías Renovables; las mismas serán implementadas en mayor número de casos buscando solución a la diversificación de la matriz energética de la República Argentina.

Se plantea la integración de Energías Renovables para lograr ampliar la posibilidad frente a los recursos petroleros (gran porcentaje de la matriz energética del país), los cuales se tiene conocimiento son de carácter no renovable.

Mediante el uso del software Homer Grid Pro (Figura 18) se conocerá cómo funcionarían las tecnologías que se planean instalar en la casa. 


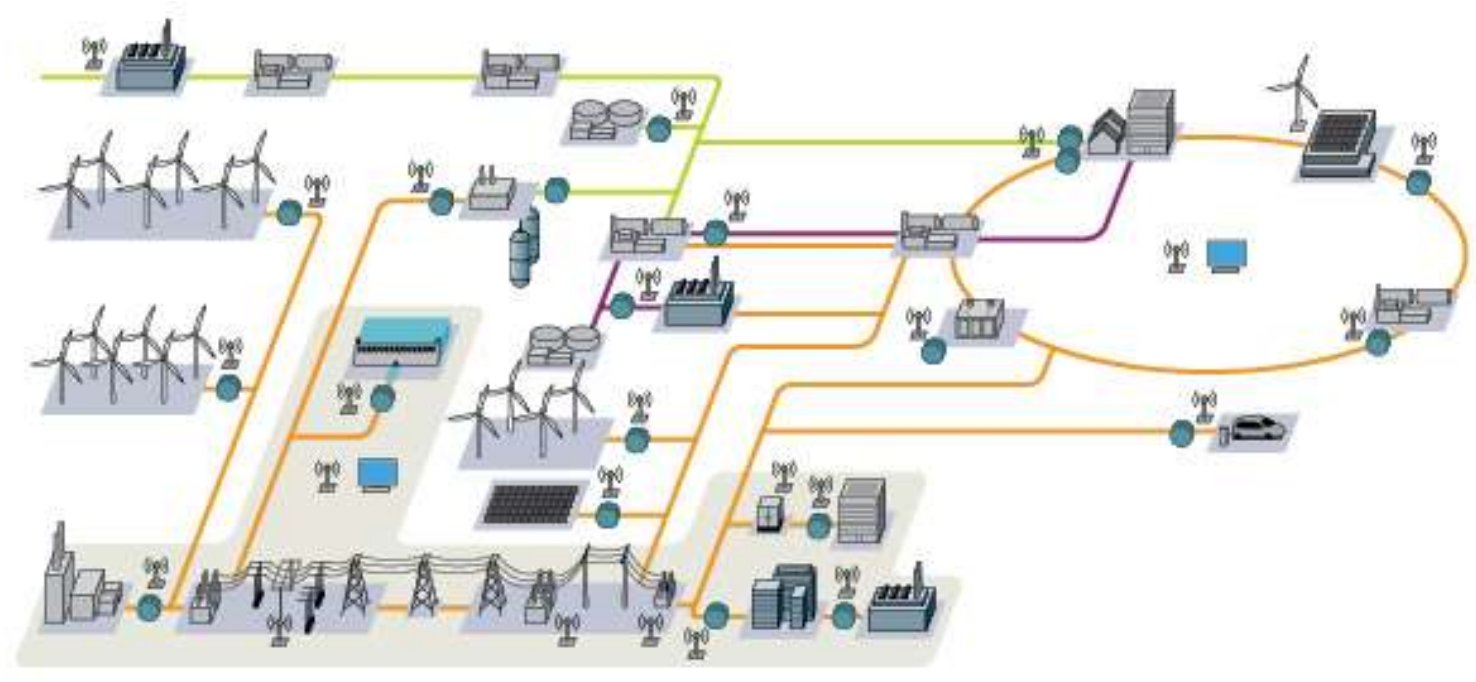

Figura 18. Ejemplo de uso de Homer Grid Pro

Como ya es conocida la eficiencia de este tipo de tecnologías a instalar en la casa-tipo y de los diferentes beneficios que representan en una locación un tanto apartada de los medios energéticos convencionales, se planea llevar a cabo este proceso de instalación.

Es a partir de este punto (ya conocidos todos los impactos que tendrá sobre la casa-tipo) que se planea todo tipo de estudios para proseguir con dicha instalación. Los estudios a realizar serán de encontrar mejores aparatos a instalar.

El presente trabajo no tiene como objetivo el reemplazo de los mismos, sino más bien el conocimiento de otro tipo de fuentes de energía que puedan abastecer energéticamente a las sociedades futuras. Se plantea de la misma manera ampliar la cultura energética de la misma, esto permitirá que la sociedad realice un uso racional de la energía.

El abastecimiento energético mediante los tipos de energías convencionales NO resulta viable debido al gran costo económico que implicarían su conexión en plaza y los costos fijos que implican los mismos y que no resultan totalmente necesarios. 


\section{REFERENCIAS BIBLIOGRÁFICAS}

Abastecimiento energético (s.f.). En Real Academia de Ingeniería. Recuperado de http://diccionario.raing.es/es/lema/abastecimiento-energ\%C3\%A9tico

Aerogenerador 1kw. (s.f.). En AEOLOS, wind turbine. Recuperado de http://www.windturbinestar.com/Aerogenerador-1kw.html

Aerogenerador Vertical Aeolos-V 1kw. (s.f.). En AEOLOS, wind turbine. Recuperado de http://www.windturbinestar.com/1kw-Aerogenerador-Vertical.html

Argentina se posiciona como el $7^{\circ}$ país en capacidad instalada eólica en Latinoamerica. ( 2 de marzo de 2018). En Asociación Argentina de Energía Eólica. Recuperado de https://argentinaeolica.org.ar/novedades/argentina-se-posiciona-como-el-7-pais-encapacidad-instalada-eolica-en-latinoamerica

Beneficios de los aerogeneradores de eje vertical. (s.f.). En Suministros y Montajes Eólicos S.L. Recuperado de http://www.e-vawt.es/index.php?seccion=beneficiosaerogeneradores

Cómo hacer un biodigestor casero. (23 de febrero de 2019.). En EcoInventos Green technology. Recuperado de https://ecoinventos.com/biodigestor-casero/

Curso de Biomasa y Biocombustible del MSc Labriola, Carlos V. M.

Derroche energético: ¿cuál es la situación actual?. (s.f.). En Rincón Educativo, Energía y Medio Ambiente. Recuperado de http://www.rinconeducativo.org/es/recursoseducativos/derroche-energetico-cual-es-la-situacion-actual

Índice de desarrollo humano. (s.f.). En Wikipedia. Recuperado de https://es.wikipedia.org/wiki/\%C3\%8Dndice_de_desarrollo_humano

Kit Panel Solar 50w Regulador 10a Bat 24amp Inversor 150w. (s.f.). En Mercadolibre. Recuperado de https://articulo.mercadolibre.com.ar/MLA-656801869-kit-panel-solar50w-regulador-5a-bateria-26amp-inversor-150w-_JM

La Argentina tiene el consumo más alto de gas por habitante en América del Sur. (16 de agosto de 2016). En Telam, Agencia Nacional de Noticias. Recuperado de http://www.telam.com.ar/notas/201608/159188-energia-consumos.html

Lampara Led 9w Interelec X 50 Foco 220v E27 10w. (s.f.). En Mercadolibre. Recuperado de https://articulo.mercadolibre.com.ar/MLA-626013474-pack-x50-lampara-led-e27-9wo-10w-fria-foco-220v-interelec- JM

Mantenimiento de tu instalación fotovoltaica. Recomendaciones para Propietarios e Instaladores. (s.f.). En SunFields, venta de Placas Solares. Recuperado de https://www.sfe-solar.com/noticias/articulos/instalaciones-fotovoltaicas-tipos-demantenimiento/

Mercadolibre (s.f.). En Mercadolibre. Recuperado de https://www.mercadolibre.com.ar/

Recurso energético (s.f.). En Wikipedia. Recuperado de https://es.wikipedia.org/wiki/Recurso_energ\%C3\%A9tico

Salamandras, calefactores o estufas a leña. (s.f.). En El Maitén. Recuperado de http://www.elmaitenmuebles.com.ar/calefaccion/salamandras/salamandras_linea_com pleta-datos.php 